Cahiers « Mondes anciens »

ANCIENS

Histoire et anthropologie des mondes anciens

5 | 2014

Maudire et mal dire : paroles menaçantes en Grèce ancienne

\title{
Paroles menaçantes et mots interdits en Grèce ancienne : approches anthropologiques et juridiques
}

Vincent Azoulay et Aurélie Damet

\section{OpenEdition}

Journals

Édition électronique

URL : http://journals.openedition.org/mondesanciens/1211

DOI : 10.4000/mondesanciens.1211

ISSN : 2107-0199

Éditeur

UMR 8210 Anthropologie et Histoire des Mondes Antiques

Référence électronique

Vincent Azoulay et Aurélie Damet, «Paroles menaçantes et mots interdits en Grèce ancienne :

approches anthropologiques et juridiques », Cahiers « Mondes anciens » [En ligne], 5 | 2014, mis en ligne le 14 février 2014, consulté le 30 avril 2019. URL : http://journals.openedition.org/ mondesanciens/1211; DOI : 10.4000/mondesanciens.1211

Ce document a été généré automatiquement le 30 avril 2019.

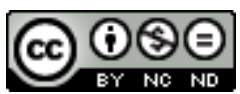

Les Cahiers «Mondes Anciens " sont mis à disposition selon les termes de la licence Creative Commons Attribution - Pas d'Utilisation Commerciale - Pas de Modification 4.0 International. 


\title{
Paroles menaçantes et mots interdits en Grèce ancienne : approches anthropologiques et juridiques
}

\author{
Vincent Azoulay et Aurélie Damet
}

1 «Sac à vin, œil de chien, cœur de cerf!» L'Iliade s'ouvre, comme chacun sait, sur un torrent d'injures proférées par Achille à l'encontre d'Agamemnon qui l'a privé de sa part de butin - la belle Briséis. Loin d'être anecdotique, ce déchaînement verbal permet de poser, en guise de préambule, une série de questions fondamentales sur le rôle des paroles menaçantes dans le monde grec archaïque et classique ${ }^{1}$.

Pour commencer, cette séquence inaugurale met en lumière la dimension processuelle du sujet. Car les paroles menaçantes n'éclatent jamais comme un coup de tonnerre dans un ciel serein, mais s'inscrivent toujours dans des cycles d'actions et de réactions plus ou moins longs. En l'espèce, si le poème commence avec la fureur (mènis) d'Achille, cette colère en renvoie à d'autres en miroir et, en premier lieu, au courroux d'Apollon, outragé par le sort réservé à son prêtre Chrysès (v. 43 et 74), dont la fille Chryséis est tenue captive par Agamemnon. C'est là le début d'un cycle infernal : tandis que la mort rôde dans le camp achéen, le devin Calchas révèle à Agamemnon les raisons de la colère divine (v. 101 sq.). Le roi se sent outragé par ces paroles de mauvais augure, qu'il interprète comme des injures faites à son honneur (timè). Saisi par la colère, Agamemnon décide alors d'humilier Achille, son meilleur combattant, en exigeant qu'il lui donne sa propre captive, Briséis, de manière à compenser le renvoi de Chryséis à son père. C'est là que vient s'intercaler le flot d'insultes lancées contre Agamemnon par Achille qui fait ensuite le « grand serment " (mega horkos) de ne plus combattre dans les rangs des Achéens. Dans cette séquence, l'insulte apparait donc comme un élément parmi d'autres dans un long cycle de "réciprocité négative », pour reprendre le vocabulaire cher aux anthropologues ${ }^{2}$ 
Cette longue séquence permet aussi de penser la contiguïté entre différentes formes de paroles menaçantes qu'il convient de ne pas séparer a priori ${ }^{3}$. Ces colères successives et emboîtées s'accompagnent en effet d'actes de langage variés qui, tous, ont pour propriété d'entretenir une spirale néfaste et mortifère : malédiction (du côté de Chrysès); insultes et serment (dans le cas d'Achille). Malédictions, serments et insultes forment ainsi un système, sans doute parce qu'ils constituent, chacun à leur manière, des réponses à des outrages préalables.

4 Si l'on se concentre sur les seules insultes, la scène iliadique permet également de réfléchir aux différentes formes que celles-ci peuvent revêtir. Outre des paroles dégradantes jouant sur l'animalisation de l'injurié (« œil de chien », « cœur de cerf ») ou stigmatisant son manque de contrôle («impudent», «lourd de vin »), l'Iliade met en scène un bien étrange type d'injures: la franchise ou, pour parler comme les Grecs de l'époque classique, la parrhèsia ${ }^{4}$. Le devin Calchas craint en effet (et à raison) de dire la vérité à Agamemnon de peur de susciter de sa part une réaction outragée (v. 74 sq.) : la franchise peut donc être considérée comme une insulte dès lors qu'elle est ressentie comme une atteinte à la timè royale. Au-delà du cas d'espèce, l'exemple montre bien qu'il n'existe aucun critère linguistique infaillible permettant de définir le seuil à partir duquel une parole se mue en insulte : seule une analyse contextuelle permet d'établir, au coup par coup, ce qui relève de l'injure et ce qui n'y ressortit pas.

L'épisode jette aussi une lumière crue sur l'inquiétant pouvoir des paroles menaçantes. Car s'il faut retenir une leçon du premier chant de l'Iliade, c'est que les mots peuvent tuer : ainsi est-ce la malédiction de Chrysès qui déclenche la vengeance d'Apollon, dont les flèches lancées sur les Achéens sont le terrible symbole de l'efficacité propre à la parole rituelle. Sur un mode atténué, les insultes ont aussi une capacité performative: l'altercation entre Achille et Agamemnon mérite à cet égard de se lire comme une réflexion sur le basculement, toujours possible, de la violence verbale à la violence physique - celle qui fait couler le sang. Nulle automaticité toutefois en la matière, car les insultes ne sont pas toujours suivies d'effets. Certes, après avoir copieusement insulté Agamemnon, Achille s'apprête à le tuer d'un coup d'épée (v. 149 sq.), comme si l'injure préparait le passage à l'acte violent; toutefois, Athéna arrête son geste au dernier moment et lui propose, comme dérivatif à sa colère, d'insulter son adversaire tout son saoul, mais de ne pas lever la main sur lui. Comme l'affirme la déesse, "venge-toi en paroles, quoi qu'il arrive» (v. 207 sq.). L'insulte sert donc ici d'exutoire, empêchant le sang de couler ${ }^{5}$. Remarquons aussi que, dans les deux cas - la malédiction de Chrysès et les insultes d'Achille - les dieux apparaissent, non comme de simples témoins, mais comme des acteurs décisifs de l'échange, soit qu'ils activent, soit qu'ils inhibent la violence contenue dans les paroles menaçantes.

Cette séquence permet enfin d'aborder la dimension proprement politique de ces actes de langage. Toutes ces paroles menaçantes sont en effet échangées lors d'une assemblée, sous le regard du laos (v. 53 sq.), et non à l'occasion d'un tête-à-tête à huis-clos. Loin d'être neutre, ce cadre collectif influe de façon décisive sur le déroulement de l'action : d'une certaine façon, c'est la présence de la communauté - même muette - qui oblige les acteurs à réagir de façon violente pour ne pas paraître accepter l'outrage public fait à leur honneur. Dès lors, cette scène permet d'aborder de front l'interrogation qui est à la source de notre enquête : quel lien établir entre la politique et les paroles menaçantes en Grèce ancienne? Les insultes sont-elles le signe d'un dérèglement civique ou, au contraire, sont-elles le reflet d'un fonctionnement politique normal? De fait, si le 
politique se définit par le conflit et l'organisation du dissensus - comme le soutient Nicole Loraux $^{6}$ - les paroles menaçantes pourraient bien faire partie intégrante du système. Mais, double effrayant de la violence physique, l'insulte peut s'avérer aussi l'antichambre du désordre séditieux, comme le souffle d'ailleurs l'étymologie : lorsqu'il apparait vers 1380 , le terme « insulte » a d'abord le sens de « soulèvement » et de « sédition ».

C'est cette tension principielle que nous souhaitons explorer dans ce volume en analysant la manière dont les Grecs donnèrent, à l'époque archaïque et classique, toute leur place aux paroles menaçantes en tant qu'élément fondamental du fonctionnement civique, et, dans le même temps, cherchèrent à encadrer son expression, pour empêcher qu'elles ne dégénèrent en stasis sanglante. En définitive, nous entendons mettre au jour une véritable civilisation de l'insulte en Grèce ancienne : si les paroles menaçantes apparaissent comme un trait de civilisation, caractéristique du fonctionnement politique grec, elles doivent néanmoins être civilisées et domestiquées, afin de ne pas mettre en péril la société tout entière.

\section{Les paroles menaçantes comme trait de civilisation}

8 L'insulte faisait à ce point partie du mode de vie des Grecs de l'époque archaïque qu'ils en avaient fait un genre poétique à part entière : la poésie iambique marquée par le blâme (le psogos), dont Ralph Rosen et Pierre Destrée présentent, dans ce volume, la facette ironique et mordante. Pendant obscur du chant de louange, les vers iambiques mettaient en forme l'agressivité verbale et étaient réputés pour leur efficacité terrifiante, puisque, selon la tradition, l'inventeur supposé du genre, Archiloque, aurait poussé au suicide les victimes de ses vers ${ }^{7}$. On tient là l'émergence d'une tradition satirique pluri-séculaire à l'intérieur de laquelle les poètes et les orateurs n'hésitaient pas à réutiliser les gestes et les armes rhétoriques de leurs prédécesseurs pour donner plus de poids encore à leurs railleries, ainsi que le montre Deborah Steiner.

Mais ces paroles menaçantes ne s'épanouissaient pas seulement dans le cadre de performances poétiques ou philosophiques, nécessairement réservées à une élite restreinte ; elles s'inscrivaient aussi au cœur du système civique, dans les prises de parole devant la communauté tout entière - ou, du moins, devant une fraction significative de la population citoyenne - sur le modèle épique de la querelle entre Achille et Agamemnon devant l'assemblée achéenne. L'insulte irrigue ainsi la comédie ancienne et les plaidoyers attiques, discours publics par excellence qui, chacun à leur façon, stigmatisent un adversaire par le medium de l'attaque verbale. Et celle-ci n'a pas toujours mauvaise presse : Isocrate, dans son Panégyrique, insiste sur l'efficacité de l'injure (loidoria) utilisée à bon escient ${ }^{8}$. Et par le procédé préféré des orateurs, la prétérition, ceux-ci font mine d'être les ennemis de l'insulte qu'ils emploient pourtant à grande échelle, la présentant toujours comme une « fâcheuse nécessité »".

10 Cette omniprésence des paroles menaçantes dans le discours public athénien conduit alors à poser le problème de la liberté de parole à Athènes. L'Athènes classique accorde en effet une valeur primordiale à l'isègoria ${ }^{10}$ (droit à la parole égal pour chaque citoyen) et de la parrhèsia (la franchise, la liberté de tout dire), fierté athénienne arborée comme un blason par Périclès en pleine oraison funèbre, histoire de rappeler l'un des nombreux traits distinctifs entre la cité attique et la cité spartiate ${ }^{11}$. Démosthène, quelques décennies plus tard, insiste sur le fait qu'à Athènes on peut encenser Sparte et dénigrer 
Athènes, alors qu'à Sparte on ne peut que louer Sparte ${ }^{12}$. Écoutons enfin Socrate plaisantant avec un certain Polos en lui soufflant qu'il « souffrirait un traitement affreux si, en venant à Athènes, le lieu de la Grèce où l'on a la plus grande liberté de parole, [il était] le seul à en être privé »"13. À Athènes, on aurait donc pu « tout dire », pour reprendre l'étymologie de la parrhèsia sur laquelle Suzanne Husson revient dans son article qui présente en miroir les pratiques socratique et cynique du franc-parler.

Ce constat reste évidemment trop général et demande à être précisé. Disons seulement, à titre provisoire, que les lieux où sont proférées les paroles menaçantes ne sont pas indifférents. Pour Aristote, il existe ainsi une différence essentielle entre le discours tenu à l'Assemblée et la diatribe prononcée au tribunal ${ }^{14}$ : les harangues devant le peuple n'insultent jamais des adversaires présents et visent des groupes de personnes hostiles à l'orateur, et non des individus précis. Devant les dikastes, il est en revanche fréquent de s'en prendre violemment et nommément à tel ou tel individu : l'attaque ad personam est la règle. Et l'auditoire, nous rappelle Eschine, a « tendance à écouter avec plaisir les insultes et les accusations $»^{15}$.

Les lieux ont également tendance à orienter le contenu même des insultes. Virginia Hunter (1990) a par exemple montré qu'un moyen efficace de dénigrer un adversaire politique dans un plaidoyer est de remettre en cause la légitimité citoyenne de la mère de l'antidikos ${ }^{16}$ ou de rappeler à l'auditoire le comportement public honteux de son père ${ }^{17}$. Reprenant ce dossier complexe, Noémie Villacèque montre ici à quel point l'injure généalogique constitue une arme de destruction rhétorique massive dans la cité athénienne.

Quant aux insultes proférées sur la scène comique - qui forment la matière de la contribution de Jean-Noël Allard - elles stigmatisent tout particulièrement le corps et l'usage déréglé qu'on peut en faire. Ce type d'attaques n'est toutefois pas le propre de la Comédie, puisqu'on les retrouve sur la scène philosophique et judiciaire - par exemple, dans l'évocation bien connue de l'androgynos chez Platon, dans le Banquet, mais aussi chez Eschine qui accuse Démosthène de porter un manteau de laine un peu trop... féminin ( Contre Timarque, 131). Et c'est précisément l'importance de ces jeux de vêtements - et la façon dont ils participent à l'énonciation de l'insulte - qui se trouve au cœur de l'article de Deborah Steiner.

Plus généralement, depuis les travaux de Pauline Schmitt Pantel (2009), on sait à quel point les comportements qu'on caractérise aujourd'hui comme " privés » ou intimes non seulement sont toisés par l'opinion publique, mais intègrent une série de normes sociales qui construisent l'image du bon ou du mauvais citoyen. On comprend alors que les insultes à caractère sexuel aient étayé le portrait de l'adversaire politique, et que l'on ait même retrouvé sur un certain nombre d'ostraka des épithètes attaquant les mœurs des citoyens que l'on souhaite bannir: "adultère" (moichos), dans le cas de Mégaclès, le second ostracisé de l'histoire ; " enculé » (katapugon), dans le cas de Thémistocle, et même " incestueux », dans le cas de Cimon ${ }^{18}$.

15 C'est également le corps de l'adversaire qui est visé par les katadesmoi, ces tablettes de malédiction, dont on connaît tant d'exemples dans le monde grec: « je te lie la langue », «je te lie les membres", " je te lie le sexe », sont des formules récurrentes dont le rituel est censé assurer l'efficacité ${ }^{19}$. En effet, l'acte énoncé est censé se répercuter sur la victime, par analogie et sympathie. On aurait tort de ne voir là que des pratiques résiduelles ou archaïques, voire de simples « affaires de bonnes femmes » : ce qui est en jeu n'est rien moins que la fabrique d'un lien indissociablement religieux et politique. 

et la communauté n'hésite pas à tenter de mobiliser, à son avantage, l'inquiétante puissance des Érinyes. Démosthène mentionne ainsi, dans son plaidoyer Contre Aristocrate (§ 97) une pratique de ce type ayant cours dans les principales institutions athéniennes: «C'est celui qui a consciemment trahi ou égaré [les juges] qui tombe sous le coup de la malédiction (enochos tèi arai); et c'est pourquoi, à chaque assemblée, le héraut maudit ( kataratai) non pas ceux qui seraient trompés, mais celui qui trompe par ses discours le Conseil ou le peuple ou l'Héliée. » C'est le héraut - émanation de la cité - qui prend en charge cette parole destinée à assurer l'euphémie, la bonne parole, et la cohésion de la communauté face au danger de la division. Malédiction et bénédiction forment à cet égard comme les deux faces d'une même médaille.

17 Toutefois, insultes et malédictions ont la fâcheuse tendance à déborder le cadre qu'on leur assigne et il arrive parfois qu'au lieu de renforcer le consensus civique, elles le rompent irrémédiablement. C'est l'une des leçons les plus aiguës de l'œdipe à Colone de Sophocle, où le personnage d'EEdipe voue ses fils à s'entretuer aux portes de Thèbes - la discorde entre frères se communiquant alors à la cité (Nancy Worman). Les malédiction ( arai) ont souvent des effets inattendus et dépassent parfois la volonté même de celui qui les profère, comme le rappelle la destinée de maints héros malheureux - CEdipe donc, mais aussi Thésée ou Amyntor, qui vouent leur progéniture à la destruction, avant de le regretter amèrement (Jean-Baptiste Bonnard). Cette terrifiante efficacité des actes de parole exige dès lors des formes de régulation qu'il convient d'étudier à parts égales. Comment les Grecs ont-ils civilisé la violence des mots pour éviter le basculement toujours possible dans la stasis?

\section{Civiliser l'insulte et les paroles menaçantes}

On aurait tort en effet de faire du monde grec, en général, et d'Athènes, en particulier, un espace libéral et libertaire où les paroles menaçantes circuleraient sans entraves. Les Athéniens ont cherché à civiliser l'insulte et à lui donner une forme qui en canalise la puissance. Cette régulation passe d'abord par un encadrement rituel qui limite la portée transgressive des injures lancées dans la comédie attique - sous l'œil vigilant de Dionysos ${ }^{20}$-et que l'on retrouve aussi dans certaines fêtes, comme la célébration des Mystères d'Éleusis. Lorsque le cortège des initiés ramène les objets sacrés (hiéra) à Athènes et traverse le Céphise, des Athéniens accueillent la procession au "pont des Plaisanteries " et abreuvent les mystes d'injures mêlées d'histoires grivoises, dont certaines ciblent nommément des hommes politiques contemporains ${ }^{21}$. L'insulte y trouve une mise en forme ritualisée qui lui donne son efficacité tout en limitant les risques de débordement.

La régulation de l'insulte se repère en outre dans les velléités de censure mise en place par le cadre "philosophique" - pour employer un mot qui recouvre en réalité des performances de nature fort diverse. On rappellera que certains penseurs, tels Xénophane, Platon ou Isocrate, ont envisagé les récits homériques et hésiodiques relatant les déchirements du panthéon divin - adultères, vols, affrontements pères/fils, gigantomachies et autres scènes conjugales - comme des blasphèmes à l'encontre des dieux, relevant de la kakègoria («mauvaise parole»), et devant ainsi être soumis à la censure. La critique présocratique et platonicienne des poèmes du haut archaïsme cherche donc à s'arroger une forme de contrôle de la parole, dans le champ particulièrement sensible qu'est celui de la piété envers les dieux ${ }^{22}$. 
20 Mais la régulation des paroles injurieuses est surtout prise en charge par la cité ellemême, par le biais de lois, de décrets et de procédures juridiques bien précises qui méritent un examen détaillé. De prime abord, si l'on s'en tient aux tirades de la comédie ancienne, il semblerait que cette dernière ne connaisse aucune entrave langagière ${ }^{23}$. L'immunité comique s'illustre notamment par le fait qu'Aristophane ne respecte pas la loi sur l'injure attribuée à Solon, telle que Plutarque la décrit, et qui est censée être en vigueur à Athènes à l'époque classique :

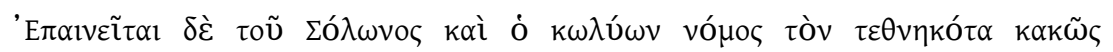



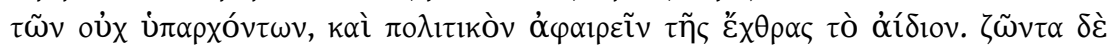

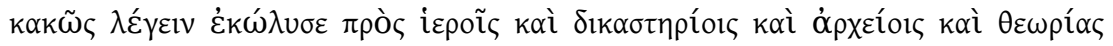

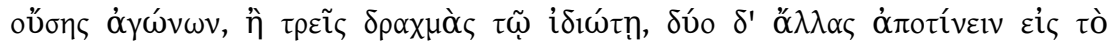

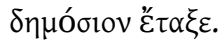

On fait aussi l'éloge de la loi de Solon qui défend de dire du mal des morts. La piété veut en effet qu'on regarde les défunts comme sacrés, la justice, que l'on ne s'attaque pas à ceux qui ne sont plus, et la politique, que les haines ne soient pas éternelles. Il défendit aussi d'injurier les vivants dans les sanctuaires, dans les tribunaux, dans les lieux où siègent les magistrats et lors de l'assistance aux concours; le contrevenant devait verser une amende de trois drachmes au particulier insulté et de deux drachmes au trésor public ${ }^{24}$.

21 L'interdiction de l'injure au mort ${ }^{25}$ est sinon bravée, du moins contournée par les comiques : Aristophane raille notamment Périclès, l'accusant d'avoir déclenché la Guerre $\mathrm{du}$ Péloponnèse, alors que celui-ci a déjà rejoint les bords du Sty ${ }^{26}$. Démosthène, dans le Contre Midias, nous renseigne aussi sur l'interdiction d'insulter les magistrats dans l'exercice de leur fonction ${ }^{27}$ :

Si on outrage ou injurie dans le privé un thesmothète, on se voit intenter une action privée en injures, publique en outrages : si c'est au contraire le thesmothète qui est

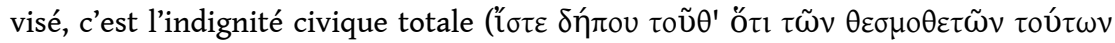

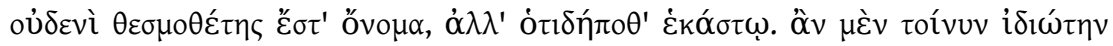

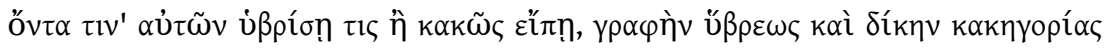

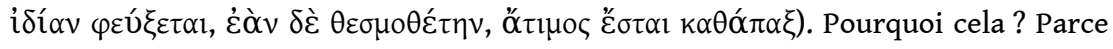
que l'homme qui agit ainsi outrage ( $\pi \rho \circ \sigma u \beta \rho i ́ \zeta \varepsilon l)$ la loi, la couronne qui est le signe de la fonction publique, le nom même de la cité; le nom de thesmothète n'appartient à personne, mais à la cité. Il en est de même pour l'archonte : si on l'injurie ou on le frappe quand il a la couronne sur la tête, c'est l'indignité civique ;

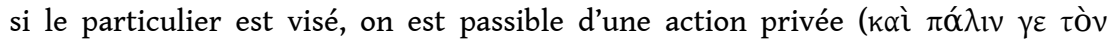

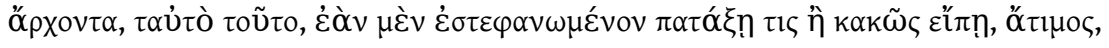

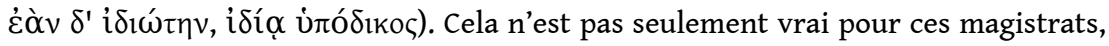
mais aussi pour tous ceux à qui la cité confère, avec l'inviolabilité, le droit de porter la couronne, ou quelque autre honneur ${ }^{28}$.

22 À supposer que le théâtre de Dionysos soit bien considéré comme un lieu public, la comédie semble ne pas être soumise à ces différentes mesures : Périclès est insulté de son vivant, notamment par Eupolis $^{29}$, et Cléon est une cible comique privilégiée par Aristophane. Ce dernier ne se prive pas en outre d'utiliser à plusieurs reprises le terme de patraloias $^{30}$ et, à l'encontre de Cléonymos, le qualificatif de "lâcheur de bouclier " ${ }^{31}$, rhipsaspis : ces deux termes appartiennent à la catégorie juridique des «mots interdits » ( aporrhèta), sur laquelle Francis Larran revient dans sa contribution. En effet, quatre injures à fin diffamatoire étaient proscrites à Athènes : patraloias, mètraloias ${ }^{32}$, rhipsaspis et androphonos ${ }^{33}$, soit quatre termes désignant le fils violent envers son père ou sa mère, le déserteur et le meurtrier. On voit ainsi poindre les notions défendues par la morale collective à Athènes: le patriotisme, piétiné par les lâches qui abandonnent le rang; la 
pureté de l'espace civique potentiellement menacée par les miasmes du meurtre; l'autorité sacrée des géniteurs. D'une façon générale, les insultes dessinent, en creux, un ensemble de normes plus ou moins explicites, qui définissent les attentes qui pèsent sur toute la société et, en particulier, sur les élites civiques. En interdisant l'emploi des termes précités, la cité délimite la sphère des valeurs qui lui importent et opère un rapprochement symbolique et politique entre criminel, mauvais enfant et mauvais patriote, trois délits qui étaient punis à Athènes de la peine de mort ou de l'atimie.

Robert Wallace (1993) pointe aussi l'analogie partielle entre ces aporrhèta et les personnes interdites de parole à l'Assemblée athénienne, et soutient que la loi sur l'insulte a été pensée pour limiter les abus langagiers, car de telles accusations verbales, même infondées, ont le pouvoir de détruire les adversaires politiques ${ }^{34}$ : élevée au rang de divinité, la rumeur a une force extraordinaire dans l'Athènes classique ${ }^{35}$. Un citoyen injurié par le biais de l'un de ces quatre termes pouvait enclencher une dikè kakègorias: elle entraînait pour l'accusé-insulteur, s'il perdait le procès, une amende de cinq cents drachmes dont on ne sait qui était vraiment le bénéficiaire final ${ }^{36}$. Chez Plutarque, on l'a $\mathrm{vu}$, mention est faite d'une amende versée en partie à l'individu insulté et en partie au trésor public: ce partage entre la collectivité et la victime implique que l'outrage consécutif aux insultes privées s'étend bien à la communautée ${ }^{37}$. Cette implication de la collectivité politique dans la condamnation de l'insulte s'explique par le fait que l'injure relève plus généralement de l'hubris, l'outrage ${ }^{38}$; elle n'est pas seulement du ressort de la kakègoria, davantage privée. D'ailleurs, le Contre Midias, juxtaposant précisément kakègoria et hubris, reflète bien cette dualité conceptuelle et juridique, dans l'Athènes classique, qui permet de traiter l'insulte soit par la procédure publique de la graphè hubreos, soit par la procédure privée de la dikè kakègorias ${ }^{39}$.

Platon, dans sa construction législative utopique, demeure tout aussi soucieux de réglementer l'emploi des injures. Le philosophe introduit cependant une autre distinction, entre la loidoria et la kakègoria ; si le terme loidorein, qui signifie injurier, n'a rien de juridique chez Platon, il n'en va pas de même de la kakègoria que le philosophe réprouve et encadre sévèrement :

Une seule loi sera instituée pour interdire à tous ceux dont je viens de parler cette conduite inacceptable: la voici. Que nul n'injurie personne. Quand dans un entretien il y a matière à contestation, chacun devra écouter les raisons de l'autre et exposer les siennes, tant à son adversaire qu'aux personnes présentes, sans y mêler aucune injure. Se répandre en imprécations les uns contre les autres, se couvrir mutuellement d'injures et se diffamer comme des mégères, ce n'est au point de départ qu'une affaire de mots, une chose légère, mais dans les faits, ils engendrent les haines et les inimitiés les plus lourdes. [...] Il sera interdit à quiconque de ne jamais rien faire entendre de pareil dans un temple, ni dans un sacrifice public, dans les concours, ni sur la place publique, ni autre tribunal ni en aucun lieu autre lieu public. [...] Ce que nous disons à présent, c'est que l'homme qui est pris dans un échange de propos outrageants est impuissant à s'abstenir de chercher en même temps à faire rire aux dépens de celui qui insulte, et c'est la forme que prend l'outrage toutes les fois que nous nous laissons emporter. Mais quoi! L'humeur des comiques, toujours prête à lancer le ridicule sur les gens, la tolérerons-nous si c'est sans colère qu'elle poursuit ce but, de tourner en ridicule nos citoyens ? [...] qu'il ne soit permis à nul auteur de comédies, de poésie iambique ou lyrique, que ce soit en paroles ou en actes, avec colère ou sans colère, de tourner en ridicule, en aucune façon, un citoyen. Si quelqu'un désobéit, les présidents des concours le chasseront du pays définitivement, le jour même, sous peine pour eux 
d'être condamnés à trois mines consacrées au dieu en l'honneur duquel se déroule le concours ${ }^{40}$ Magnésie, il est interdit à tout auteur de comédie, de poésie iambique ou lyrique de tourner en ridicule, en paroles ou en actes, un citoyen, au risque d'être tout simplement banni. Ce n'est pas la première fois que Platon se révèlerait plus draconien que la législation attique.

Pour autant, Athènes était certainement plus tatillonne en la matière que ce que l'on a parfois pu suggérer. Revenons à ce propos à la question épineuse de la censure appliquée au genre comique, qui semble s'épancher en toute impunitée ${ }^{41}$. Si Stephen Halliwell (1991) soutient que les lois athéniennes sur la diffamation ne concernaient pas la satire comique, Max Radin (1927) avait auparavant montré l'évolution de la terminologie insultante chez Aristophane, notamment celle du terme «rhipsaspis ». Dans trois pièces datant d'avant 420, Aristophane qualifie expressément Cléonymos de lâcheur de bouclier, insulte exprimée selon plusieurs tournures ${ }^{42}$. Dans les Oiseaux, datant de 414, il utilise cependant des périphrases plus subtiles, indiquant que Cléonymos a jeté son bouclier ${ }^{43}$. Max Radin émet ainsi trois hypothèses : la loi sur les aporrhèta à Athènes aurait pu être instituée entre 420 et 414 ; jusqu'à la période $420-414$, la comédie aurait pu bénéficier d'un régime d'exception, lui permettant d'utiliser des termes interdits, mais cette liberté d'expression lui aurait été retirée ; l'insulte liée à l'abandon du bouclier sur le champ de bataille aurait pu être ajoutée à la liste des aporrhèta entre 420 et 414 , ce qui pourrait s'expliquer par le contexte tendu de l'expédition de Sicile. La politique s'invite donc dans la réflexion: l'encadrement de l'insulte n'est pas seulement une question purement juridique, mais dépend des circonstances de la vie politique athénienne.

S'il existe des possibilités légales de rétorsion aux insultes, celles-ci sont ainsi loin d'être automatiquement activées, et ce en fonction des contextes, plus ou moins propices aux principes de l'isègoria et de la parrhèsia. Notre Cléonymos ainsi raillé et traité de déserteur aurait pu poursuivre Aristophane par le biais d'une dikè kakègorias. Mais un tel procès n'aurait probablement pas assagi la verve mordante d'Aristophane, si l'on se réfère aux vaines attaques judiciaires initiées par Cléon contre le dramaturge. En effet, Cléon aurait, selon certains passages des Acharniens, entamé une procédure contre Aristophane car ce dernier se serait moqué du peuple athénien dans une de ces productions ${ }^{44}$, les Babyloniens. Bien que son intrigue soit difficile à reconstituer, cette pièce semble avoir instruit le

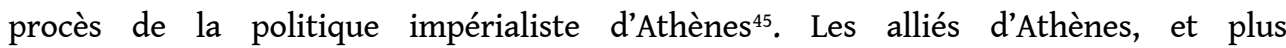
particulièrement les Samiens, auraient été injuriés car comparés aux esclaves du Grand Roi, sous les traits des Babyloniens ${ }^{46}$. Cette pièce aurait pu fragiliser la ligue de Délos déjà marquée par la révolte de Mytilène en 428-427, alors qu'Athènes était en guerre contre Sparte. Il semblerait que Périclès lui-même ait instauré en 440-438, en plein siège de Samos, un décret visant à interdire les attaques sur la politique extérieure et sur le peuple, décret levé trois ans plus tard. Le même principe se vérifie ici : c'est parce que le contexte géopolitique est tendu que la censure est brièvement instaurée, et non en raison d'une loi intangible qui régulerait, une fois pour toute, les débordements langagiers.

Le cas même de Périclès permet de penser l'écart entre les procédures judiciaires prévues pour punir les insultes et leur application réelle. Le stratège fut, en effet, plus qu'à son tour victime d'insultes, sans jamais engager la moindre poursuite, ni même trahir le moindre agacement. Cette impassibilité apparaît en pleine lumière, en 430 av. J.-C., quand les Athéniens le rendirent responsable des nombreux malheurs qui les frappaient. Comme 
l'indique Plutarque, à la suite de Thucydide, "Périclès resta insensible à toutes ces attaques et supporta calmement et en silence l'impopularité et la haine $»^{47}$. Loin d'être dictée par les circonstances, cette impassibilité correspond à une stratégie délibérée de la part du chef athénien qui observe cette ligne de conduite, non seulement à l'Assemblée, mais aussi sur l'Agora dans les échanges de la vie quotidienne. Plutarque rapporte à cet égard un épisode particulièrement saisissant :

Un jour, par exemple, un individu particulièrement vulgaire et grossier l'insulta et l'accabla d'outrages. Périclès le supporta en silence, toute la journée, en pleine agora, en continuant à régler les affaires urgentes. Le soir, il s'en alla tranquillement, tandis que l'autre le suivait et lui lançait toutes les injures possibles. Avant d'entrer chez lui, comme il faisait déjà nuit, il ordonna à l'un de ses serviteurs de prendre une lampe pour raccompagner l'homme et le reconduire chez lui ${ }^{48}$.

Plutôt que de réagir comme le ferait n'importe quel citoyen, Périclès reste donc impavide et refuse de se départir de son calme, malgré les outrages répétés. Selon une forme de réciprocité négative, face à ce torrent d'insultes, la logique aurait été de répondre à l'affront en retournant la pareille ou bien de porter l'affaire devant les tribunaux, puisque, comme on l'a vu, les injures aux magistrats en fonction étaient passibles de lourdes peines ${ }^{49}$. Non seulement le stratège s'abstient de répliquer par la violence ou le droit, mais il choisit de répondre à l'humiliation par un bienfait - selon une forme de réciprocité positive - en faisant reconduire le malotru à son domicile.

Grandeur d'âme de Périclès? Peut-être, mais sans doute faut-il aussi y voir un calcul intéressé de la part du « grand homme » qui juge politiquement plus intéressant de ne pas activer les procédures légales prévues en ces circonstances. Périclès exerce en effet sur le peuple une séduction politique d'autant plus forte qu'il refuse de tirer parti de sa position de magistrat pour engager des poursuites judiciaires. Son absence de réaction face à l'insulte participe dès lors à construire sa statue d'homme d'État désintéressé.

31 Le mutisme de Périclès nous amène finalement à clore ce panorama de la logorrhée injurieuse en pays attique par l'évocation du silence. De l'interdit à l'indicible, l'insulte est à la croisée de la liberté de parole (l'aspiration à tout dire) et la crainte devant l'efficacité des mots (la tentation de ne rien dire). Les travaux de Silvia Montiglio (2000) ont montré la tension permanente, dans les sources de l'Athènes classique, entre la valeur éminente $\mathrm{du}$ logos et de la parrhèsia et, en même temps, l'importance du silence et des contournements langagiers comme stratégie d'euphémisation. On comprend dès lors à quel point l'usage des injures est imbriqué dans une codification extrême qui attache une importance fondamentale au pouvoir magique du langage. Louis Gernet considérait ainsi qu'aporrhètos, comme arrhètos, était bien l'équivalent latin de nefandus, " abominable, qui ne devrait pas être prononcé ", à cause de ce dangereux pouvoir d'évocation de termes liés à un tabou ${ }^{50}$. Il y a ainsi dans la liste juridique des aporrhèta étudiée précédemment une indéniable dimension symbolique. C'est donc ce prisme juridico-anthropologique qui permet de saisir toute la portée du laborieux dialogue entre Tirésias et đEdipe, à qui le devin tente de faire comprendre, sans la dévoiler, l'identité du meurtrier qu'il recherche ${ }^{51}$ . Inceste, meurtre, parricide ${ }^{52}$, đdipe a commis l'arrhètos et l'aporrhètos, et nous rappelle à quel point l'injure peut aussi mener à l'ineffable. 


\section{BIBLIOGRAPHIE}

Azoulay V. (2010), Périclès, la démocratie à l'épreuve du grand homme, Paris.

Azoulay V. et Boucheron P. (2009), « Les violences intellectuelles, nouvel objet d'histoire », dans Azoulay V. et Boucheron P. éd., Le mot qui tue. Les violences intellectuelles de l'antiquité à nos jours, Paris, p. 23-52.

Bremmer J. (2000), « Verbal insulting in ancient Greek culture », AAntHung 40, p. 61-72.

Brenne S. (1994), «Ostraka and the Process of Ostrakophoria », dans Coulson W. D. E. et al. éd., The Archaeology of Athens and Attica under Democracy, Oxford, p. 13-24.

Brockmann C. (2003), Aristophane und die Freiheit der Komödie. Untersuchen zu den frühen Stücken unter besondere Berücksichtigung der Acharner, Munich.

Carrière J.-C. (2000), « Les comédies perdues d'Aristophane ", dans Jouanna J. éd., Le théâtre grec antique : la comédie, Cahiers de la villa Kérylos 10, Paris, p. 197-236.

Clay D. (1982), « Unspeakable words in Greek tragedy », AJPh 103, p. 277-298.

Csapo E. et Slater W. (1995), The context of ancient drama, Ann Arbour.

Damet A. (2012), La septième porte. Les conflits familiaux dans l'Athènes classique, Paris.

Demont P. (2006), « Hubris, outrage, anomie et démesure de Gernet à Fisher », dans BrilletDubois P. et Parmentier É. éd., Philologia, mélanges offerts à Michel Casevitz, Lyon-Paris, p. 347-359.

Detienne M. (1982), « La Rumeur, elle aussi, est une déesse », GH 5, p. 71-80.

Foucault M. (2008), Le Gouvernement de soi et des autres. Cours au Collège de France 1982-1983, Paris.

Fisher N. (1992), Hybris : a study in the values of honour and shame in Ancient Greece, Warminster.

Gager J. G. (1992), Curse Tablets and Binding Spells from the Ancient World, Oxford.

Gernet L. (1958), La répression de l'injure verbale en droit attique (tapuscrit, Bibliothèque GernetGlotz).

Gernet L. (2001), Recherches sur le développement de la pensée juridique et morale en Grèce, Paris ( $1^{\mathrm{e}}$ éd. 1917).

Giordano M. (1999), La parola efficace. Maledizioni, giuramenti e benedizioni in Grecia antica, PiseRome.

Glotz G. (1899), « Kakègorias dikè », dans Daremberg C. et Saglio É. éd., Dictionnaire des Antiquités grecques et romaines, t. III, 1, Paris, p. 788-791.

Gotteland S. (1997), « La rumeur chez les orateurs attiques : vérité ou vraisemblance », AC 66, p. 89-119.

Halliwell S. (1991), « Comic satire and freedom of speech in classical Athens », JHS 111, p. 48-70.

Halliwell S. (2000), «Le rire rituel et la nature de l'Ancienne Comédie Attique », dans Desclos M.-

L. éd., Le rire des Grecs : anthropologie du rire en Grèce ancienne, Grenoble, p. 155-168.

Harrison A. R. W. (1968), The Law of Athens, Oxford. 
Hunter V. (1990), « Gossip and the politics of reputation in classical Athens », Phoenix 54, p. 299-325.

Larran F. (2011), Le bruit qui vole. Histoire de la rumeur et de la renommée en Grèce ancienne, Toulouse.

Lenfant D. (2003), « Des décrets contre la satire : une invention de scholiaste ? Pseudo-Xén., II, 18, schol. Ach. 67, schol. Av. 1297 », Ktéma 28, p. 5-31.

Loraux N. (1997), La cité divisée. L'oubli dans la mémoire d'Athènes, Paris.

MacDowell D. (1978), The law in classical Athens, New York.

Mélèze-Modrzejewski (1998), « Paroles néfastes et vers obscènes », Dike 1, p. 151-169.

Montiglio S. (2000), Silence in the Land of Logos, Princeton.

Moreau A. (1979), « À propos d'đモdipe. La liaison entre trois crimes, parricide, inceste et cannibalisme », Ét. de litt. anc., Paris, p. 97-127.

Orfanos C. (1998), « L'épisode du parricide dans les Oiseaux d'Aristophane », Pallas 48, p. 119-134.

Parker R. (1983), Miasma : pollution and purification in early Greek religion, Oxford.

Radin M. (1927), « Freedom of speech in ancient Athens », AJPh 48, p. 215-230.

Saetta Cottone R. (2005), Aristofane e la poetica dell'ingiuria : per una introduzione alla loidoria comica, Rome.

Scafuro A. (2001), « Parents abusers, military shirkers, and accused killers : the authenticity of the second law inserted at Dem. 24.105 », dans Gagarin M. et Wallace R. W. éd., Symposion, Vienne, 2001, p. 51-69.

Scheid É. (2005), « Remarques sur les fondements de la vengeance en Grèce archaïque et classique », dans Bertrand J.-M. éd., La violence dans les mondes grec et romain, Paris, p. 395-410.

Schmitt Pantel P. (2009), Hommes illustres. Hommes illustres. Mours et politique à Athènes au ve siècle, Paris.

Sommerstein A. H. (2004), " Harassing the Satirist : the alleged attempts to prosecute Aristophanes ", dans Sluiter I. et Rosen R. M. éd., Free Speech in Classical Antiquity, Leyde, p. $145-174$.

Svenbro J. (1985), « Vengeance et société en Grèce archaïque. À propos de la fin de l'Odyssée », dans Verdier R. et Poly J.-P. éd., Vengeance, pouvoirs et idéologies dans quelques civilisations de l'Antiquité, Paris, 1985, p. 47-63.

Wallace R. W. (1993), « The Athenian laws against slander », dans Thür G. éd., Symposion 1993. Vorträge zur Griechischen und Hellenistische Rechtsgeschichte, Cologne, p. 109-124.

Wallace R. W. (2005), « Law, Attic Comedy, and the Regulation of Comic Speech », dans Gagarin M. et Cohen D. éd., The Cambridge Companion to Ancient Greek Law, Cambridge, p. 357-373.

\section{NOTES}

1. Ce travail prend sa source dans un colloque international organisé les vendredi 30 et samedi 31 mars 2012, à l'Institut national d'histoire de l'art, avec le soutien de l'Institut universitaire de France et des équipes de recherche Analyse comparée des pouvoirs (ACP) et Anthropologie et histoire des mondes antiques (ANHIMA).

2. Svenbro 1985 ; Scheid 2005. 
3. Giordano 1999. Manuela Giordano revient sur ces thèses dans ce volume en comparant la relation entre injures et vengeance en contexte albanais contemporain et en contexte attique.

4. Sur la question de la mise en danger de celui qui « dit vrai », propre à la notion de parrhèsia, voir les belles réflexions de Michel Foucault 2008.

5. Voir sur cette question Azoulay et Boucheron 2009.

6. Loraux 1997.

7. Pindare, Pythique, II, 55.

8. Isocrate, Panégyrique, 130.

9. Montiglio 2000, p. 29.

10. Démosthène, Contre Aphobos, 3 ; Platon, République, 557b ; Gorgias, 461e ; Euripide, Hippolyte, v. 422 ; Ion, v. 679-675, Suppliantes, v. 430-442 ; Phéniciennes, v. 392 ; Oreste, v. 917-922 ; Isocrate, Paix, 14. Voir Wallace 1993, p. 109 ; Wallace 2005.

11. Thucydide, II, 37, 2.

12. Démosthène, Contre Lochitès, 106.

13. Platon, Gorgias, 461e.

14. Aristote, Rhétorique, III, 1418a, 27.

15. Eschine, Sur la couronne, 3-4.

16. Sur la remise en cause du statut d'une mère comme athénienne: Eschine, Sur l'ambassade infidèle, 78, 93, 180 ; Démosthène, Sur la couronne, 129-131; Sur l'ambassade, 281 ; Contre Midias, 149-150 ; Contre Aristogiton I, 65 ; Contre Euboulidès, 30-37, 40-45.

17. Démosthène, Contre Androtion, 33-34, 56, 58, 68 ; Contre Timocratre, 125, 127, 168.

18. Brenne 1994.

19. Voir par exemple Gager 1992.

20. Saetta Cottone 2005.

21. Strabon, IX, 1, 24; Hésychius, s.v. Gephyrismos et gephyristai (femme/homme lançant des insultes aux citoyens éminents ou aux passants). Voir aussi les Thesmophories et l'échange d'obscénités entre femmes (Diodore de Sicile, 5.4.7). Sur le rire rituel, voir Halliwell 2000.

22. Sextus Empiricus, Contre les physiciens, IX, 193 (Xénophane B 11 D.K) ; Sextus Empiricus, Contre les philologues, I, 289 (Xénophane B 12 D.K.) ; Isocrate, Busiris, 38-41 ; Platon, République, 378b-c. Voir Damet 2012, p. 378-390.

23. C'est la position de Stephen Halliwell (1991, p. 49) qui conclut que les lois athéniennes sur la diffamation ne concernaient pas la satire comique.

24. Plutarque, Vie de Solon, 21, 1-2. Dans Aristophane, Guêpes, v. 1206-1207, il est question d'une poursuite judiciaire en cas de loidoria, injure. Voir aussi Démosthène, Contre Aristocrate, 50 ; Contre Midias, 32. Sur la dikè kakègorias voir Glotz 1899; Gernet 1958; Harrison 1968, p. 67 et 155; MacDowell 1978, p. 127 ; Clay 1982 ; Parker 1983, p. 132 ; Wallace 1993 ; Mélèze-Modrzejewski 1998 ; Bremmer 2000 ; Montiglio 2000, p. 135-137 ; Scafuro 2001 ; Damet 2012, p. 332-338.

25. Rappel de l'interdiction d'insulter les morts : Démosthène, Contre Lochitès, 104 ; Contre Boeotos II, 49 ; Plutarque, Vie de Solon, 1. Rappel de l'interdiction d'insulter les vivants : Plutarque, Vie de Solon, 21, 1 ; Platon, Lois, 935b-c.

26. Aristophane, Acharniens, v. 530-534 et Aristophane, Paix, v. 606-611.

27. Lysias, Pour le soldat, 6.

28. Démosthène, Contre Midias, 32-33.

29. Eupolis, fr. 102K.

30. Aristophane, Nuées, v. 991 et 1327 ; Oiseaux, v. 1337 ; Grenouilles, v. 274 et v. 773. Voir Orfanos 1998 ; Damet 2012, p. 79-89.

31. Sur l'évocation de Cléonymos comme un «lâcheur de bouclier ", voir Aristophane, Guêpes, v. 19 et 592 ; Paix, v. 678 et 1297 ; Nuées, v. 353 et Oiseaux, v. 287 et 1473.

32. Lysias, Contre Theomnèstos, 6, 8; Isocrate, Contre Lochitès, 3. Il existait peut-être d'autres insultes incluses à la liste des aporrhèta, comme d'accuser quelqu'un de travailler sur l'agora, de 
se prostituer ou d'avoir dilapidé l'héritage paternel, si l'on établit un lien entre les personnes interdites de parole à l'Assemblée et les questions posées lors de la docimasie. Voir Damet 2012, p. 404-408.

33. Sophocle, Edipe-Roi, v. 362. Tirésias rappelle qu'il est interdit, indicible, arrhèton, d'appeler quelqu'un « meurtrier ».

34. Wallace 1993, p. 119.

35. Detienne 1982 ; Hunter 1990 ; Gotteland 1997 ; Larran 2011.

36. Isocrate, Contre Lochites, 3 : «Les législateurs ont jugé si graves les coups et blessures qu'ils

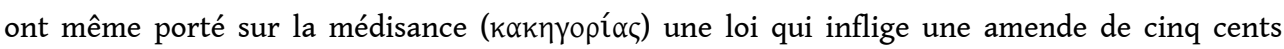
drachmes à ceux qui disent quelque chose qui doit rester caché ».

37. Louis Gernet (1958) penche aussi pour le partage entre cité et initiateur: "malgré la restriction de ce délit, l'État, tout de même, la société dans son ensemble lui voit une certaine signification et y reconnaît quelque chose de punissable ».

38. Fisher 1992 ; Demont 2006.

39. Démosthène, Contre Midias, 32.

40. Platon, Lois, 934e-935b.

41. Wallace 1993, p. 116.

42. Aristophane, Paix, v. 678 ; Nuées, v. 353 ; Guêpes, v. 19 et 592.

43. Aristophane, Oiseaux, v. 287-289 et 1473-1481.

44. Csapo et Slater 1995, p. 170-171. Voir aussi Lenfant 2003 ; Sommerstein 2004 ; Brockmann 2003 ; Azoulay 2010, p. 25.

45. Sur cette pièce, voir Carrière 2000, p. 205-207

46. Aristophane, Babyloniens, fr. 71 K.-A. Les Samiens se sont révoltés contre Athènes en 440 ; les prisonniers ont été marqués comme des esclaves.

47. Plutarque, Périclès, XXXIV, 1. Cf. déjà Thucydide, II, 60, 1.

48. Plutarque, Périclès, $\mathrm{V}, 2$.

49. Démosthène, Contre Midias, 32-33.

50. Gernet 2001, p. 238. Voir aussi l'étude de Silvia Montiglio (2000, p. 37) qui montre en quoi arrhètos et aporrhètos finissent par se confondre et être synonymes, dans la sphère légale et sacrificielle.

51. Sophocle, Edipe-Roi, v. 350-362. Tirésias appelle Oedipe, meurtrier de son père, «souillure impie de ce pays » et, devant l'incompréhension tenace du roi sacrilège, il finit par employer le terme d'androphonos, mais soigneusement scindé en début et en milieu de vers. Voir Clay 1982.

52. Moreau 1979.

INDEX

Mots-clés : injure, insulte, malédiction, dérision, Grèce ancienne, droit grec, comédie

Keywords : insult, imprecation, Ancient Greece, mockery, Greek law, greek tragedy 


\section{AUTEURS}

\section{VINCENT AZOULAY}

Université Paris-Est Marne-la-Vallée, ACP - EA3350

\section{AURÉLIE DAMET}

Université Paris-1 Panthéon-Sorbonne, ANHIMA - UMR8210 